\title{
Attention and Target Selection for Smooth Pursuit Eye Movements
}

\author{
Vincent P. Ferrera and Stephen G. Lisberger \\ University of California at San Francisco, Department of Physiology, W. M. Keck Foundation Center for Integrative \\ Neuroscience, and Neuroscience Graduate Program, San Francisco, California, 94143
}

\begin{abstract}
Two rhesus monkeys were trained to track a small moving target in the presence of a moving distractor. The target and distractor were distinguished by their color. Smooth pursuit eye movements were quantified in terms of the latency of the eye movement and the open-loop eye acceleration profile. Smooth pursuit latencies for single targets were on the order of $100 \mathrm{msec}$. When the target was paired with a distractor moving in the same direction as the target, pursuit latencies decreased to roughly $85 \mathrm{msec}$. When the target was paired with a distractor moving in the opposite direction, pursuit latencies increased to roughly $150 \mathrm{msec}$. The motion of the distractor had no significant effect on the eye acceleration profile. Experiments were performed to dissociate visual search for the target from pursuit initiation by providing a spatial cue rather than the color cue. These experiments showed that visual search necessarily preceded pursuit initiation only when the distractor moved in the opposite direction relative to the target. In this case, visual search contributed about $25 \mathrm{msec}$ to the overall latency of pursuit. Control experiments showed that the monkey need not attend to the distractor in order for it to influence the latency of pursuit. A network model was developed in which units that represent the motions of the target and distractor compete against one another. Attention serves to bias the outcome of this competition toward the direction of the selected target. The performance of this network exhibits a striking parallel to the effect of the distractor on smooth pursuit latency.
\end{abstract}

[Key words: smooth pursuit, sensorimotor bottleneck, competitive networks, eye movements, visual attention, macaque]

Primates, including humans, make two types of voluntary eye movements: short, quick saccades to stationary targets, and slower, more sustained smooth pursuit of moving targets. Smooth pursuit depends upon retinal image motion and functions to stabilize the retinal image of small objects that move at slow to moderate speeds. When there is only one moving target, the smooth pursuit system acts much like a visuomotor reflex that matches eye velocity to target velocity (see Lisberger et al.,

\footnotetext{
Received May 22, 1995; revised July 7, 1995; accepted July 13, 1995

We especially thank Dr. Karl Gegenfurtner for providing us with the software libraries needed to run our graphics display. We also thank J. Schwart $z$ and $A$ Tartaglia for technical assistance. This work was supported by the McDonnellPew Program in Cognitive Neuroscience Fellowship JSMF 92-38 to V.P.F and NIH Grant EY0.3878 to S.G.L.

Correspondence should be addressed to Dr. Vincent P. Ferrera, Department of Physiology. P.O. Box (0444, University of California at San Francisco, San Francisco, CA 94143.

Copyright 1995 Socicty for Neuroscience $0270-6474 / 95 / 157472-13 \$ 05.00 / 0$
}

1987). When there is more than one potential target, some selection or decision process must precede the initiation of pursuit. The decision to select a target before the initiation of an eye movement represents a constriction of information that we refer to as the sensorimotor bottleneck. The bottleneck occurs because there is more information represented in the multiple target mo. tions entering the system than there is in the single eye movement trajectory that is emitted. Here, we report behavioral observations on monkeys that were trained to choose between two possible smooth pursuit targets presented simultaneously on a video monitor. These observations support a model wherein the neural signals arising from the potential targets compete in a winner-take-all network. The role of attention in this framework is to bias the outcome of the competition in favor of the selected target.

The basic neuroanatomical substrate for smooth pursuit has been mapped by a combination of anatomical experiments (Brodal, 1978, 1979, 1982; Fries, 1981; Glickstein et al., 1980, 1985; Gerrits and Voogd, 1989), physiological recordings (Suzuki and Keller, 1984; Bruce et al., 1985; Mustari et al., 1988; Newsome et al., 1988; Thiers et al., 1988; Stone and Lisberger, 1990), and lesion studies (Zee et al., 1981; Newsome et al., 1985; Dursteler et al., 1987; Lynch, 1987; Dursteler and Wurtz, 1988; Suzuki et al., 1988; Keating, 1991; MacAvoy et al., 1991). Visual inputs are processed in the geniculo-cortical pathway and are transmitted from the primary visual cortex and the extrastriate visual cortex through the dorsolateral pontine nucleus (DLPN) and the cerebellum to the brainstem. The projection from the extrastriate cortex to the pons includes separate, parallel outputs from the middle temporal area (MT) and the medial superior temporal area (MST). Areas MT and MST are part of a pathway through visual cortex that is primarily concerned with spatial and motion information (Zeki, 1978; Maunsell and Van Essen, 1983; Albright, 1984) and is thought to be involved in visual orienting and guidance (Ungerleider and Mishkin, 1982; Andersen, 1987; Goodale and Milner, 1992). Much of the previously cited evidence points to MT and MST as key areas for processing the visual motion inputs to the pursuit system.

In addition to the motion pathway, there is a parallel pathway through visual cortex that comprises visual areas of the temporal lobe, is concerned with color and form information (Gross et al., 1972; Zeki, 1978; Desimone et al., 1985; Tanaka et al., 1986; Desimone and Schein, 1987; Schein and Desimone, 1990), and is thought to be involved in object recognition. The selection of targets for eye movernents provides an opportunity to study quantitatively the interactions between these two pathways. At the most abstract levels of processing, the decision of what target to pursue is probably based on attributes such as color and shape that uniquely identify the target. In other words, what one is 
aware of during the cxccution of a voluntary, goal-directed eye movement is the intention to look at a particular object, rather than the intention to move the eyes along a given trajectory. The position and movement of the target are, in a sense, incidental properties that are only necessary to specify the metrics of the eye movement. Nevertheless, when a smooth pursuit target is selected on the basis of an attribute such as color, this information must be linked with information regarding the motion of the target. One idea that fits nicely with the parallel pathways notion is that there are separate representations, or "feature maps," for color and motion. The activities in these different maps may be bound together through visual spatial attention (Treisman, 1977; Triesman and Gelade, 1980). This implies that when there are competing targets, one must attend to the location of the desired target before initiating an eye movement. One of the goals of the current study was to investigate the role of visual spatial attention (visual search) in several experiments that were designed to reduce uncertainty about the location of the target. We found that visual search does play a role in the disambiguation of competing inputs, but we would characterize this role as modulatory. That is, the latency of smooth pursuit shows a characteristic pattern depending upon whether the potential targets compete or cooperate. Attention is capable of modulating this basic pattern, but is not responsible for generating it.

In this study, we have examined the behavior of a sensorimotor pathway in the primate that is responsible for producing a smooth eye movement in response to a moving visual target. Our goal was to describe the behavior of this pathway when faced with a simple decision about which of two moving targets to use as its input. Our observations address two issues related to target selection. First, what sort of mechanism might be involved in implementing the decision to track one target or the other? Second, what is the role of attention in relation to this decision-implementing machinery? We believe that the decision is implemented by a nonlinear competitive-cooperative network and that we have identified a behavioral correlate of this competition. By manipulating the cognitive requirements of the task, we found that the competition happens automatically and does not depend on the need to attend both targets. Attention is necessary to determine the outcome of the competition, and the strength of the attentional signal can modulate the performance of the network. However, even a very weak attentional signal can give rise to an all-or-none overt response due to the selfreinforcing properties of the network.

\section{Materials and Methods}

Experiments were conducted on two male rhesus monkeys (Macaca mulatta), weighing approximately $6 \mathrm{~kg}$ each. Our methods were approved by the UCSF Institutional Animal Care and Use Committee. Monkeys were trained to move voluntarily from their home cage to a primate chair. A method modified from Wurtz (1969) was used to train each monkey to attend a stationary target. Surgery was then performed under sterile conditions to implant a coil of wire on one eye (Judge et al., 1980) and to secure a platform to the skull for head restraint (Miles and Eighmy, 1980). For all subsequent training and experintents, the monkey's head was secured to the ceiling of the primate chair and a set of field coils was lowered over the chair so that we could use the magnetic search-coil method to monitor horizontal and vertical eye position. The eye coil was calibrated by having the monkey attend to targets at different positions and the monkey was subsequently required to keep the direction of gaze within 2-30 of target position. Correct performance of the task was rewarded with drops of juice or water.

Rehavioral tasks. Monkeys were trained to track moving targets presented on a CRT monitor. We used a step-ramp target motion paradigm to minimize the occurrence of saccades during pursuit initiation (Rash- bass, 1961). Trials were initiated by requiring the monkey to look at a stationary central fixation light. After a short interval, a moving parafoveal target appeared. At the same time, the central fixation light was turned off and the monkey was required to track the target by initiating a smooth pursuit eye movement. All targets moved horizontally at vertical positions $3^{\circ}$ above or below the horizontal meridian. The initial horizontal target position was set so that targets moving towards the vertical meridian would cross it after $100 \mathrm{msec}$. The paths of two targets never crossed. The monkey was given a liquid reward, provided that he kept his gaze directed toward the desired target for the duration of the trial. The monkey's performance was monitored by tracking his eye position relative to $\mathrm{a} \pm 3.0^{\circ}$ fixation window centered around the target. Several steps were taken to ensure that the monkeys did not make anticipatory eye movements. First, the initial target location relative to the fixation mark (up, down, right, left) was randomized from trial to trial. Second, the direction of target motion (towards or away from the vertical meridian) was also randomized. Third, the time at which the target was presented or started to move was randomized. Fourth, trials were aborted if the monkey initiated an eye movement before the fixation light went off. Finally, catch trials were interleaved with normal trials. During a catch trial, the target(s) appeared as usual but the fixation light was never turned off and the monkey was rewarded only if he continued to look at the fixation light.

The basic task was one in which the monkey selected a pursuit target based on a color cue. Variations on this task will be described as they come up in the results. In the "color-cue" task (Fig. 1), the monkey initially fixated on a small $\left(0.4^{\circ}\right)$ white square in the center of the screen. After a few hundred milliseconds, the white fixation mark was replaced by a colored square (red or green) of the same size. This was the cue. After a second time interval (the stimulus onset asynchrony, or SOA), two moving targets appeared (one red, one green), one $3^{\circ}$ above and the other $3^{\circ}$ below the horizontal meridian. The monkey's task was to pursue the target that matched the color of the cue. On some randomly interleaved trials, only a single target appeared so that we could measure the animal's "normal" pursuit latency under similar visual conditions. On trials with multiple targets, the color of the cue and the position and direction of both targets were randomized so that the monkey could not anticipate the direction of the required eye movement. Trials were randomized within blocks so that the monkey was required to complete exactly one trial of each type before proceeding to the next block of trials.

Visual stimulation. Pursuit targets were generated and controlled by a Univision Piranha video framebuffer with an on-board microprocessor (Texas Instruments TMS 34020). The output from the video board was displayed on a calibrated $20^{\prime \prime}$ color monitor (Barco) with a $60 \mathrm{~Hz}$ noninterlaced refresh rate. This frame rate is perfectly adequate for elicity normal smooth pursuit (Logan and Lisberger, unpublished observations). The monitor stood at a viewing distance of $30^{\prime \prime}$ so that the display area subtended roughly $30^{\circ}$ horizontally by $20^{\circ}$ vertically. The spatial resolution of the display was 1280 pixels by 1024 lines, and the depth was 8 bits/pixel. Pursuit targets were small $\left(0.9^{\circ}\right)$ colored squares presented on a uniform gray background

The video board was programmed to send out digital pulses (frame sync) for timing purposes at the beginning of each frame in which a target was turned on or started to move. These pulses were sampled by the computer and stored along with the eye movement data. The amount of time that elapsed between the frame sync pulse and the appearance of a target on the screen can be calculated by allowing a drawing speed of $15.6 \mu \mathrm{sec}$ per raster line. We verified this by digitizing the frame sync pulse along with the output of a photovoltaic transducer (Radio Shack) that measured the luminance near the middle of the screen as a white target was drawn there on a dark background. The elapsed time depended mainly on the vertical position of the target and was about 6 msec for targets appearing ahove the initial fixation point and about 10 msec for the targets appearing below. As we generally combined data for targets above and below the fixation point, we corrected by subtracting $8 \mathrm{msec}$ from all bchavioral latency measurements. This correction is irrelevant for measurements of eye acceleration.

Eye movement recording. Eye position was monitored using at monocular scleral search coil system (CNC Engineering). Separate horizontal and vertical eye position signals were fed through an analog differentiator (lowpass, $-3 \mathrm{~dB}$ at $25 \mathrm{~Hz}$ ) to yield horizontal and vertical eye velocity. The eye position and eye velocity signals were then digitally sampled by computer at $1 \mathrm{kHz} /$ channel and stored on disk for 
A
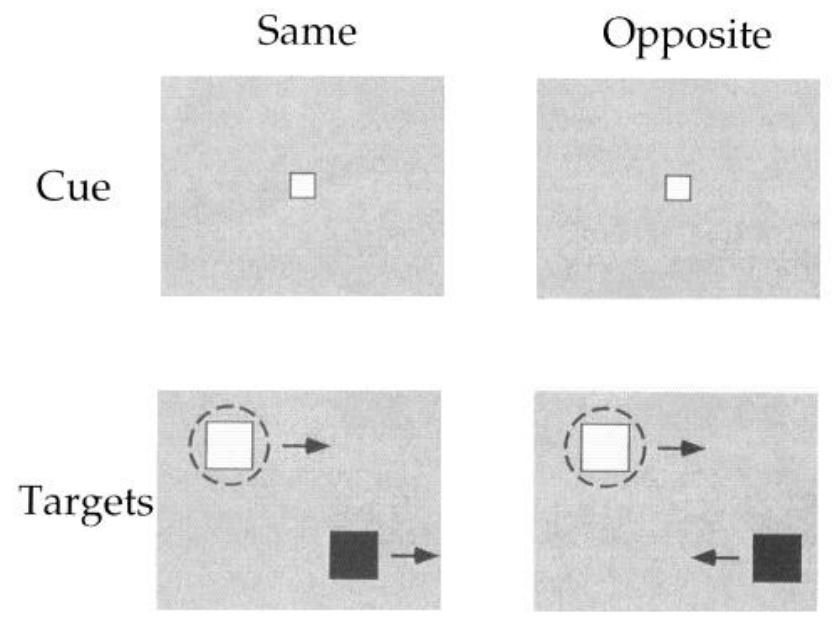

B

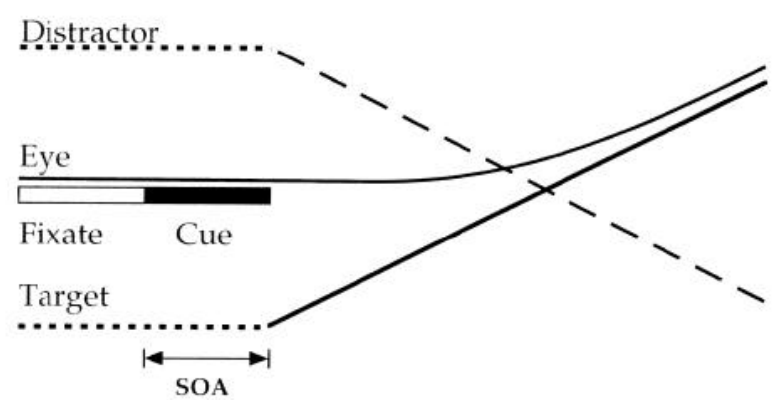

Figure 1. Illustration of the basic color-cue task that the monkeys were trained to perform. $A$, Snapshots of the video display at two instants in time for two distractor conditions. At the start of each trial, the animal foveated a white central fixation target. Shortly thereafter, the fixation target turned to one of two colors, red or green (top panels labeled cue). After another brief interval, the cue disappeared and two colored moving targets appeared. The animal's task was to look at the target that matched the color of the cue, which he did by initiating a smooth eye movement in the appropriate direction. The other target is referred to as the "distractor." The target and distractor were also separated vertically, $3^{\circ}$ above or below the initial fixation position. Two conditions are shown, one in which the target and distractor moved in the same direction, and one in which they moved in opposite directions. In both cases, the target and distractor started out in opposite hemifields. On other trials, the target and distractor started in the same hemifield. For each trial, we selected at random the color of the target, its direction (towards or away from the fovea), and its initial starting position (up, down, left, right). $B$. The same task unfolded in time for the case in which the target and distractor move in opposite directions. The horizontal axis is time and the vertical axis is horizontal eye and target position. The time between the onset of the cue and the onset of the target is the "Stimulus Onset Asynchrony" ( $S O A)$, and was varied between 100 and $800 \mathrm{msec}$.

further analysis. Only trials in which initial target motion was toward the vertical meridian were saved for analysis.

Average eye velocity records were constructed from eye velocity traces for individual trials by aligning each trace with the onset of target motion. Catch-up saccades were removed from the eye velocity traces before averaging, and eye velocity was linearly interpolated between the beginning and end of the saccade. Saccade latencies averaged around $200 \mathrm{msec}$. Latencies for smooth eye movements were estimated visually from the eye velocity records for individual trials. Eye velocity measurements are extremely sensitive to the onset of eye movement so that latencies can be assigned with a high degree of accuracy. The accuracy of our latency measurements can be seen in Figure 5, where we have constructed average eye velocity records by aligning the individual traces on the marks that we placed to indicate the beginning of pursuit. If we had systematically under- or overestimated the latency of pursuit, then one would expect to see the averages start to deviate from zero velocity before time zero. We used this method to refine and spot check our criteria for assigning pursuit latencies.

To determine eye acceleration, we first aligned eye velocity traces for individual trials on the initiation of pursuit. We then averaged the eye velocity traces for all trials that had the same target color and direction. For quantitative comparisons, we computed the average eye acceleration during successive $20 \mathrm{msec}$ intervals following pursuit initiation. The average eye acceleration over a given interval is equivalent to the difference between the final eye velocity and the initial eye velocity divided by the length of the interval. All data points for both latency and acceleration are based on averages of 10 to 40 repetitions of each trial type. Trials were excluded from the analysis if the animal selected the wrong target (as judged by the direction of the vertical saccade), initiated pursuit in the wrong direction, or failed to initiate pursuit prior to the first saccade. Smooth pursuit latencies and eye accelerations obtained with stimuli presented on a video monitor are quantitatively comparable to responses obtained with continuous stimuli projected on a tangent screen (Lisberger and Westbrook, 1985). Any artifacts produced by the refresh rate of the monitor are not evident either in the eye velocity measurements themselves or their Fourier power spectra.

\section{Results}

Target selection by color

For the first set of experiments, we trained monkeys to select a target on the basis of its color. We presented simultaneously two pursuit targets that moved horizontally but were also separated vertically. When the animal saw the targets, he selected the one that matched the color of the cue and initiated horizontal smooth pursuit with a latency generally in the range of 80 to $160 \mathrm{msec}$. He also executed a vertical saccade to foveate the chosen target, but this occurred substantially later, with latencies averaging around $200 \mathrm{msec}$. The basic pattern of results for this experiment is illustrated in Figure 2, which shows average eye velocity records from the subset of trials in which the correct target was a red square moving rightward and towards the vertical meridian (shown as a solid square in the figure). The left-most trace corresponds to the condition where the red target was paired with a green distractor moving in the same direction. The right-most trace corresponds to the condition where the red target was paired with a green distractor moving in the opposite direction. The middle trace is the eye velocity response to the red target by itself. Pursuit latencies were shortest when the distractor moved in the same direction $(78 \mathrm{msec})$, and longest when the distractor moved in the opposite direction $(162 \mathrm{msec})$. The response to the single red target had an intermediate latency (94 $\mathrm{msec}$ ). These results indicate that when the animal has to make a decision about which direction to track, pursuit initiation is substantially delayed. On the other hand, when there is only one target, or two targets moving in the same direction, there is no decision required because the desired eye velocity is the same, regardless of which target is selected. In these cases, pursuit is initiated with latencies on the order of 80 to $100 \mathrm{msec}$, although two targets produced latencies that were significantly shorter than those for single targets. The average latency of the corrective saccades did not vary with distractor condition. Furthermore, when we switched from a pursuit task to a saccade task by simply eliminating target motion, we found no difference between saccade latencies for single and double targets.

A complete experiment consisted of trials in which target color, starting location, and direction of motion were varied ran- 


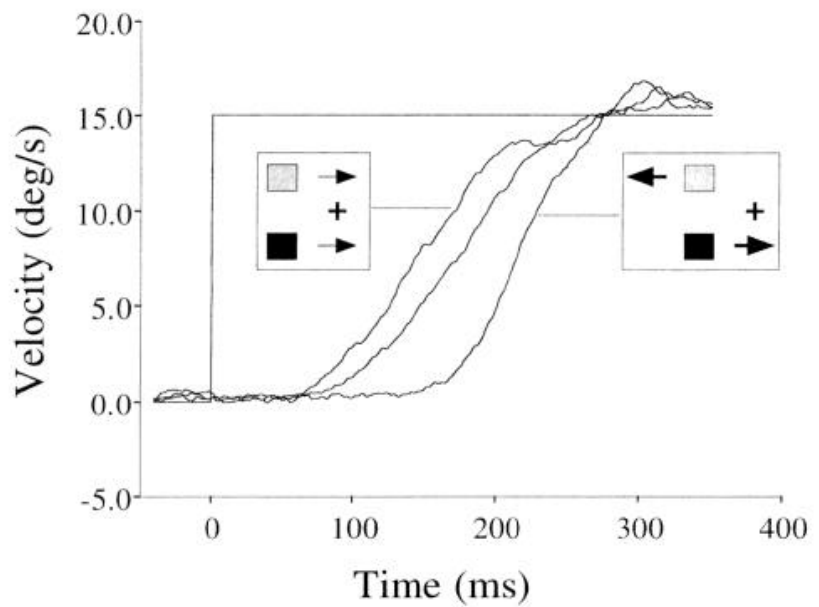

Figure 2. Effect of a moving distractor on the initiation of smooth pursuit. The three traces show averaged eye velocity for three trial types selected from a single experimental session on one monkey. For all three trial types, the target was a red square (shown as a solid square in the diagrams) moving rightward (toward the vertical meridian) at $15.0 \% \mathrm{sec}$. The left-most eye velocity trace corresponds to the condition in which the target was paired with a green distractor moving in the same direction. The right-most trace is for the condition in which the target was paired with a green distractor moving in the opposite direction. The middle trace is the eye velocity response to a red target with no distractor. The average latencies for these three conditions were 78 , 94 , and $162 \mathrm{msec}$, respectively. Traces were aligned on the onset of target motion. Target velocity is indicated by a step that starts at time zero.

domly from trial to trial. Also varied were the direction of motion of the distractor relative to the target (same or opposite) and whether the distractor starting position was in the same visual hemifield as the target or in the opposite hemifield. The latencies for two complete experiments on different monkeys are summarized in Figure 3. For each monkey there are three set of bars that reflect the distractor conditions (filled bars, single target; open bars, same direction for target and distractor; gray bars, opposite directions for target and distractor). Within each of the three main distractor conditions, the data are further separated according to target direction, color, and whether the starting positions of the target and distractor were in the same or opposite.

The average latencies for "single," "same," and "opposite" distractor conditions are shown in the row labeled "color cue" in Table 1. The basic pattern of results is that latencies for single targets averaged around $100 \mathrm{msec}$. Latencies for the "same" condition were about $15 \mathrm{msec}$ shorter than those for single targets, while latencies in the "opposite" condition were roughly $50 \mathrm{msec}$ longer. A one-way ANOVA revealed that the effect of distractor condition was significant at the $p<0.0001$ level for both monkeys. Separate one-way ANOVAs were also run for target color, direction, and hemifield. In one monkey, the effect of target direction was significant at the $p=0.01$ level. There was no significant effect of target color or hemifield for either monkey. The experiment was repeated for SOAs ("stimulus onset asynchrony;" the time between the onset of the cue and the onset of the targets) of 100, 250, 400, and $800 \mathrm{msec}$. There was no significant effect of SOA on pursuit latency for either monkey.

The experiment was repeated for speeds of $5,10,15$, and 20 dps; target and distractor speed were always equal. In Figure 4, we have plotted the average pursuit latency as a function of

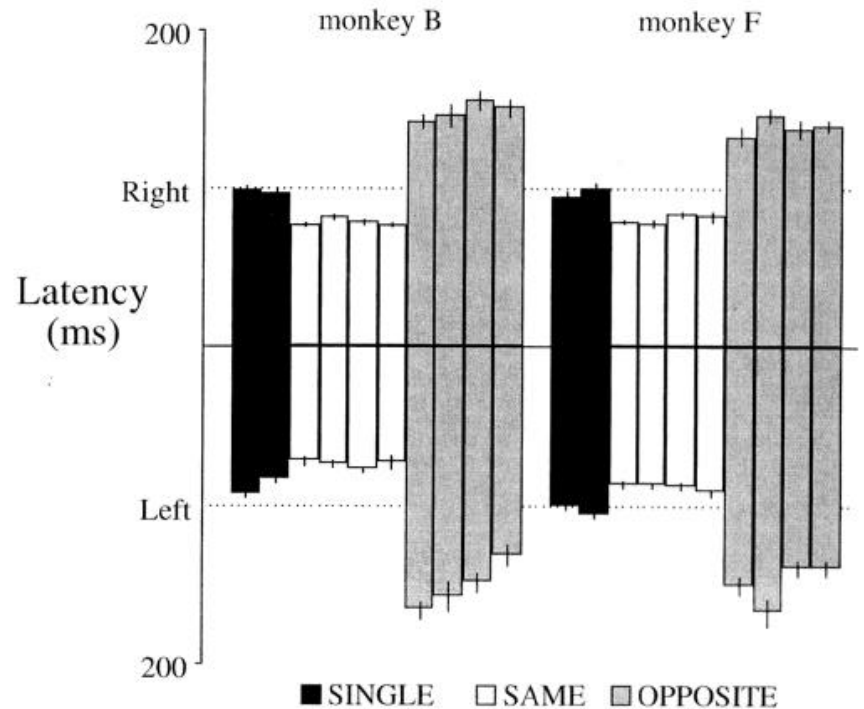

Figure 3. Smooth pursuit latencies for two monkeys sorted as a function of target direction and distractor condition. The latencies for rightward motion are plotted as bars going up, leftward as bars going down. Black bars, no distractor; open bars, distractor moving in same direction; gray bars, distractor moving in opposite direction. Latencies were also sorted according to target color and the hemifield relationship of the target and distractor (same or opposite). Thus, for each monkey, there were 10 conditions $\times 2$ directions. Reading left to right, the 10 conditions are: 1 . single green target, 2 . single red target, 3 . green target/ red distractor, same direction, same hemifield, 4. green target/red distractor, same direction, opposite hemifields, 5. red target/green distractor, same direction, same hemifield, 6. red target/green distractor, same direction, opposite hemifields, 7. green target/red distractor, opposite direction, same hemifield, 8. green target/red distractor, opposite direction, opposite hemifields, 9. red target/green distractor, opposite direction, same hemifield, 10. red target/green distractor, opposite direction, opposite hemifields. Error bars are \pm 1 SEM. The dotted lines represent $100 \mathrm{msec}$.

speed for both monkeys. Latencies were sorted by distractor condition, but all other conditions (target color, direction, and hemifield) have been collapsed into a single average. The SOA was $250 \mathrm{msec}$ for all speeds. There was a general tendency for latencies to become somewhat shorter with increasing speed. However, within this overall trend, there was still a clear and consistent effect of distractor condition.

From Figure 2 one gets the impression that longer latencies are correlated with faster accelerations, as if the eye must somehow catch up after being delayed. To look at this more closely, we analyzed eye acceleration in the following manner. First, we averaged together eye velocity traces for all trials of a particular type, aligning each trace on the onset of pursuit rather than the onset of target motion. Figure 5 shows the eye velocity traces from Figure 2 aligned in this manner. We then divided the first $80 \mathrm{msec}$ of pursuit into four time intervals of $20 \mathrm{msec}$ each and computed the average eye acceleration within each $20 \mathrm{msec}$ interval. Finally, we combined acceleration estimates for the four SOAs that were tested and for targets that appeared above and below the horzontal meridian. Figure 6 shows averaged eye acceleration in each of the four time intervals sorted in the a same manner as the latency data in Figure 3 (filled symbols, single targets; open symbols, target-distractor same direction; shaded symbols, target-distractor opposite direction). For each condition there are two data points-one for each monkey. Each point is the mean of eight average acceleration estimates (four SOAs $\times$ 
Table 1. Average smooth pursuit latencies

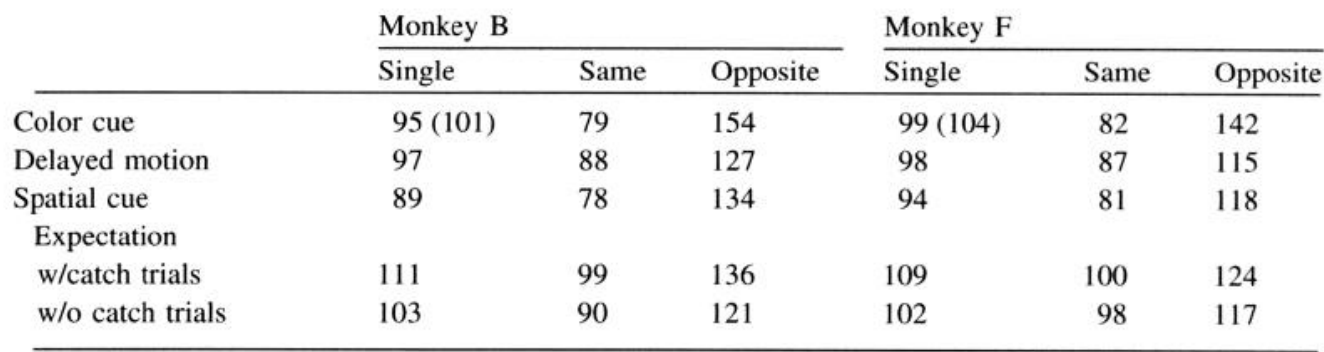

two vertical target positions) and the error bars are \pm 1 SEM of those eight measurements.

There was a good deal of variability in the acceleration data, as can be judged by the size of the error bars. To a first approximation, the motion of the distractor had little effect on the eye acceleration profile (one-way ANOVA on distractor condition, $p>0.7$ for all intervals, data separated by animal and horizontal target direction). In particular, there is no evidence that eye acceleration in any interval was slower when there was a distractor moving in the opposite direction than when the distractor moved in the same direction, as would be expected if eye acceleration were related to some vectorial combination of the target and distractor velocities. If anything, eye acceleration was slightly faster when the distractor moved in the opposite direction. This is particularly evident in the $60-80 \mathrm{msec}$ time interval. There are other manipulations, e.g., going from high to medium target contrast, for which acceleration gets faster as latency gets longer, perhaps due to integration of velocity signals during the latent interval (Lisberger and Westbrook, 1985). It seems reasonable

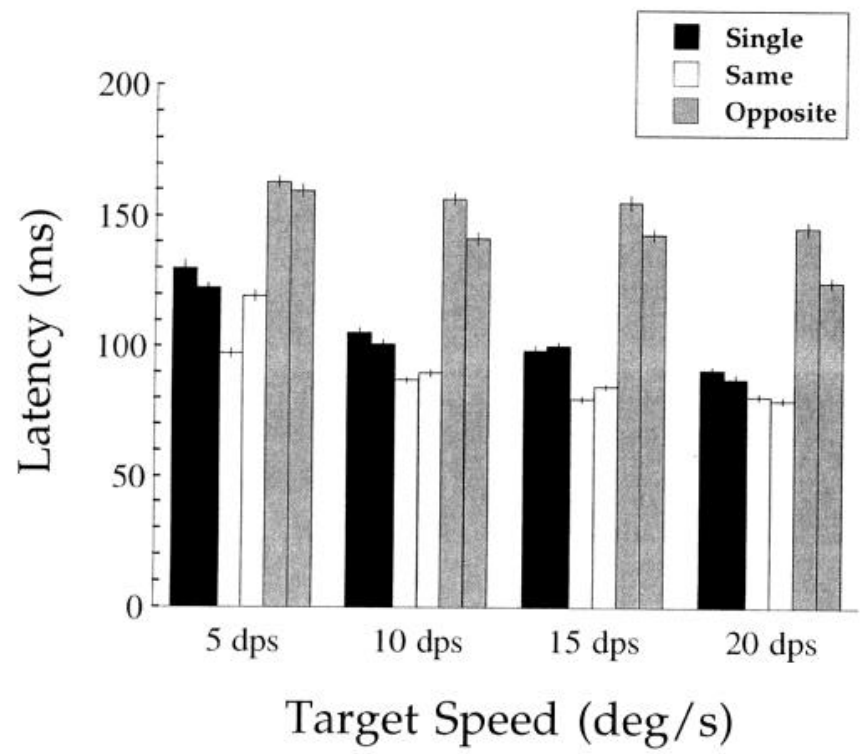

Figure 4. Smooth pursuit latencies for the color-cue task repeated at speeds of $5,10,15$, and $20 \% \mathrm{sec}$. The SOA for all speeds was $250 \mathrm{msec}$. Latencies are sorted by distractor condition, black bars, no distractor; open bars, distractor moving in same direction; gray bars, distractor moving in opposite direction. All other conditions (target color, direction, hemifield) have been collapsed into a single average. The left-most bar of each pair is the average latency for monkey $\mathrm{B}$, and the rightmost, monkey $\mathrm{F}$. The error bars represent \pm 1 SEM and are smaller than in Figure 3, as each bar represents the average of four to eight times as many trials. to conclude that the small effect of the distractor motion on eye acceleration is secondary to the much more substantial effect on the latency of pursuit.

\section{Dissociation of visual search from pursuit initiation}

In the previous task, the targets began to move as soon as they appeared on the video monitor. This means that the animal needed to search for the target that matched the cue as well as determine its direction of motion during a single brief time interval. In the next experiment, we sought to temporally dissociate visual search from pursuit initiation. We did this by having the targets appear in place at the same time as the cue, but delaying the onset of motion for 100 to $800 \mathrm{msec}$. Thus, the animal had an interval of 100 to $800 \mathrm{msec}$ in which to view both the cue and the targets and to locate the matching target. During this time, the animal was required to keep looking at the fixation target until it disappeared and the targets began to move. It should be noted that this manipulation is identical to the "motion-onset-delay" or MOD studied by Krauzlis and Lisberger (1994). That study revealed that pursuit latencies for single targets got shorter with increasing MOD. It was subsequently found, however, that this effect is obtained only in dark-adapted monkeys (S. G. Lisberger and J. D. Schwartz, unpublished observations). In the present study, we found no effect of MOD on single target latencies with background room illumination of $10 \mathrm{~cd} / \mathrm{m}^{2}$.

When the animal was given time to locate the target before

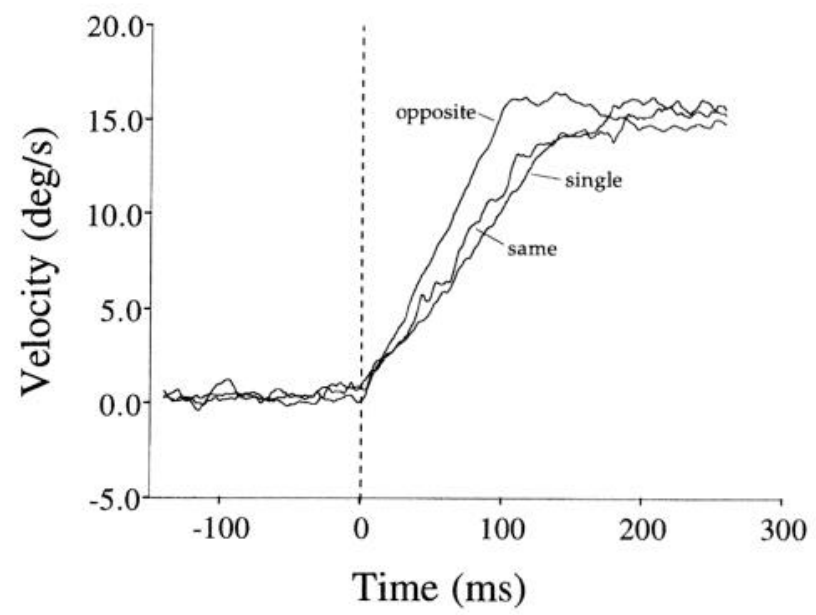

Figure 5. Averaged eye velocity as a function of time for three trial types selected from a single experimental session. For all three trial types, the target was a red square moving rightward at $15.0 \% \mathrm{sec}$. The onset of pursuit was marked on each individual trial and then all traces for a given trial type were aligned on this mark before averaging. 


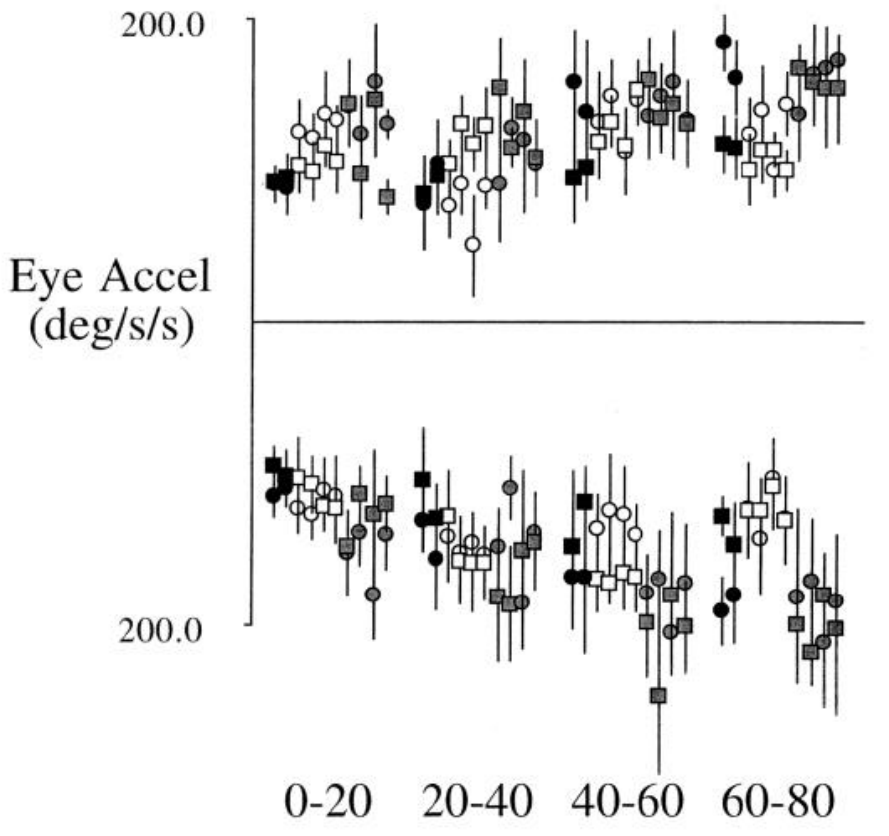

Figure 6. Average eye acceleration during four $20 \mathrm{msec}$ intervals starting at the onset of pursuit. Within each interval, accelerations are sorted by direction and by distractor condition. Black symbols, single targets; open symbols, distractor moving in the same direction; gray symbols, distractor moving in the opposite direction. Accelerations are further sorted by target color and the hemifield relationship of the target and distractor, yielding a total of 10 conditions as described in the legend for Figure 3. Square symbols are eye accelerations for monkey B, circles are for monkey F. Error bars represent \pm 1 SEM.

it started moving, the average pursuit latencies for the "same" and "single" distractor conditions were essentially the same as for the previous experiment, but latencies for the "opposite" condition were shorter by an average of $27 \mathrm{msec}$ for both monkeys (Fig. 7 and Table 1, "delayed motion"). This may be taken as a fairly direct measurement of the amount of time that the visual search process, or more precisely, that spatial uncertainty adds to the latency of pursuit under these conditions. Again, the differences between distractor conditions were highly significant (one-way ANOVA, $p<0.0001$ ). In addition, there were significant effects of target color and direction of motion for both monkeys (one-way ANOVA, $p<0.01$ ), but these differences were small, in the range of 3 to $7 \mathrm{msec}$. We also varied the SOA, although, in this case, what we are calling SOA is really the difference between when the targets appeared and when they began to move. Each monkey showed a significant effect of SOA at the $(p<0.0001)$ level, but the effect was small and inconsistent. For one monkey, the longest SOA resulted in pursuit latencies that were $5 \mathrm{msec}$ longer than those for the shortest SOA, while for the other monkey, the longest SOA corresponded to latencies that were $5 \mathrm{msec}$ shorter.

It is important to note that only one SOA was used during a given experimental session so that it was possible for the monkey to learn to use the interval during which the targets were visible but stationary as a timing cue. One might argue that this alone would have reduced the latency of pursuit relative to the original experiment in which the targets appeared moving. There are two observations that rule out this explanation. First, the same timing cue is available in the original experiment because there was a fixed duration between the appearance of the cue and the appearance of the targets. Second, one monkey (B) was

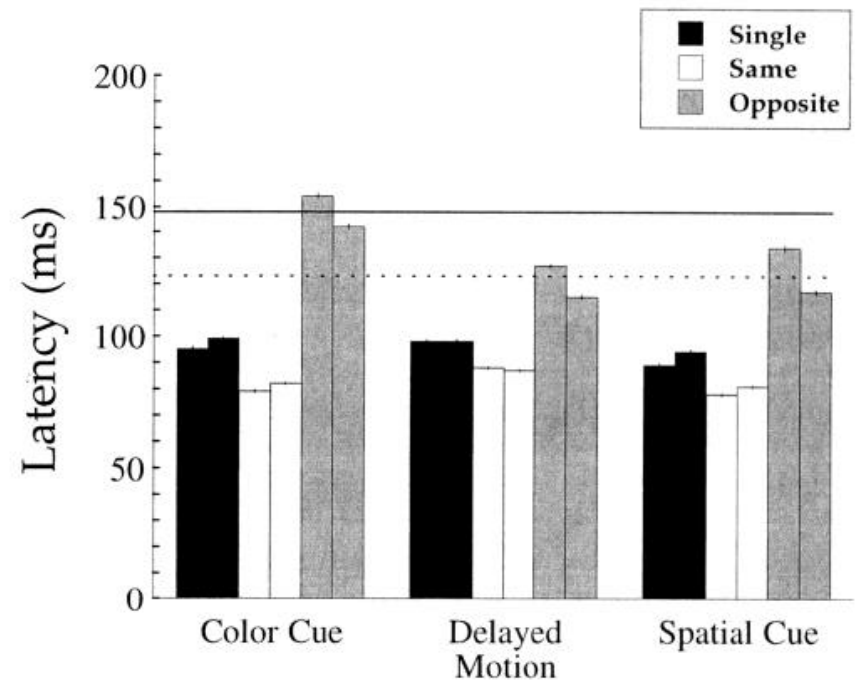

Figure 7. A comparison of smooth pursuit latencies for the color-cue task and variants of the task in which (1) the monkey was allowed time to view the cue and targets together and to locate the matching target ("delayed motion") and (2) the monkey was given an explicit cue indicating the location of the target ("spatial cue"). Latencies are sorted by distractor condition and monkey. Black symbols, single targets; open symbols, distractor moving in the same direction; gray symbols, distractor moving in the opposite direction. The left-most bar of each pair is the average latency for monkey B, and the right-most, monkey F. All other conditions (target color, direction, hemifield) have been collapsed into a single average. The solid horizontal line is the mean latency of both monkeys for the color-cue task, opposite condition $(148 \mathrm{msec})$. The dashed line is the mean latency of both monkeys for the opposite condition of both the delayed-motion and spatial-cue tasks $(123 \mathrm{msec})$. The error bars represent \pm 1 SEM and may be smaller than in Figure 3 , as each bar represents the average of many more trials.

run in an additional "delayed motion" experiment with randomly interleaved SOAs of 100, 200, 400, or $800 \mathrm{msec}$. His average smooth pursuit latency for the distractor opposite condition was $125 \mathrm{msec}$. Therefore, uncertainty about the time at which the targets began to move did not increase the latency of pursuit.

In the previous two experiments, the animal selected a pursuit target by matching the color of the target to that of the cue. In the next experiment, we sought to dissociate visual search from pursuit initiation in a way that would bypass the color-matching process altogether. We did this by eliminating the color cue and replacing it with a spatial cue (Fig. 8). In this set of experiments, the initial fixation mark never changed color. Instead, a small white spot $\left(0.4^{\circ}\right.$ square, $\left.65 \mathrm{~cd} / \mathrm{m}^{2}\right)$ was flashed in the location of the target just before the fixation mark disappeared and the targets appeared. When the targets appeared, they were moving. The targets were still of different colors, although their color was no longer relevant to the task. The spatial cue never occurred in the location of the distractor, i.e., it was a "valid" cue on $100 \%$ of the trials.

When the animal was given an explicit spatial cue, the pattern of results was much the same as when he was given time to find the matching target before it began to move (Fig. 7 and Table 1, "spatial cue"). Again, the effect of distractor condition was highly significant (one-way ANOVA, $p<0.0001$ ). Latencies for the distractor opposite condition were 20-25 msec shorter for a spatial cue than for the original color cue experiment, but still about $25 \mathrm{msec}$ longer than the single-target condition. This experiment also addressed a minor problem in comparing the previous "delayed-motion" experiment (targets are initially sta- 

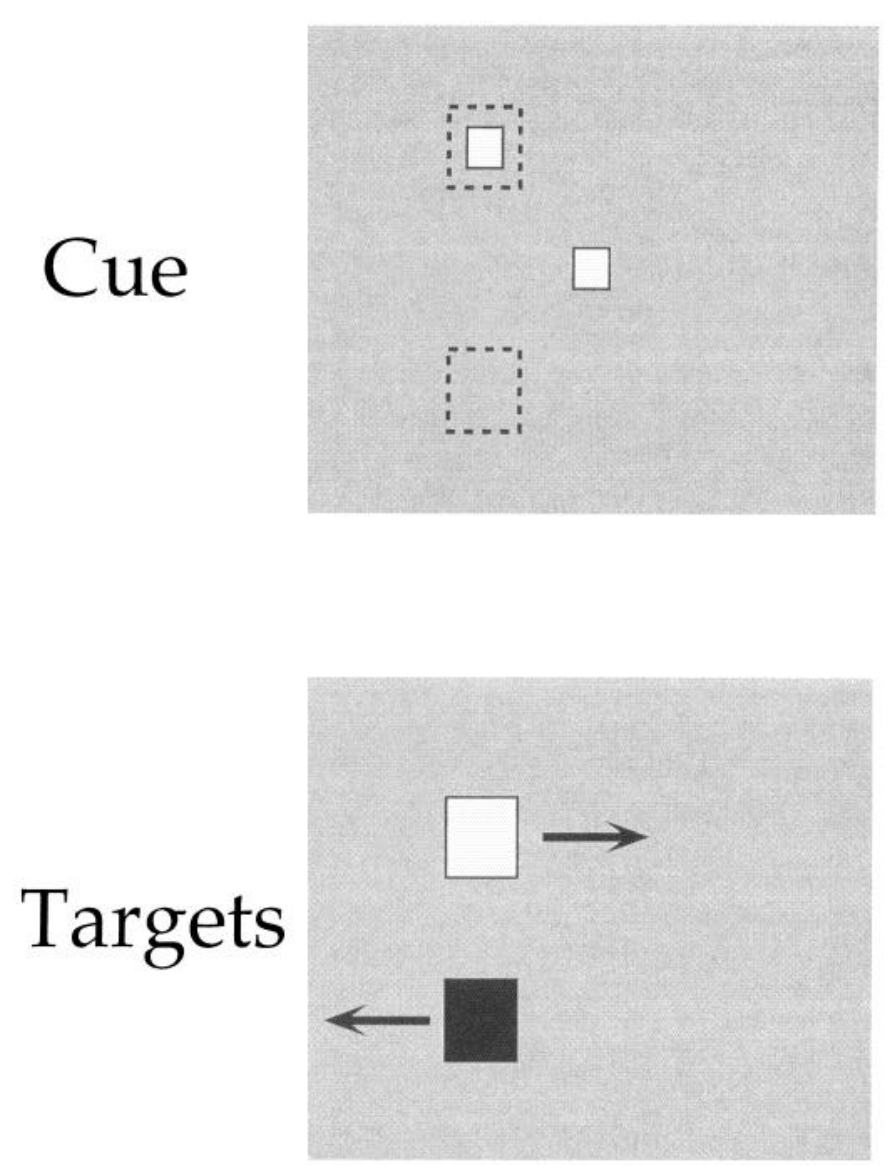

Figure 8. Schematic representation of the spatial-cue task. Initially, the monkey fixated the white target in the center of the screen (top). While he fixated, a cue was flashed in one of the possible target locations (represented by dashed boxes). A short while later (bottom), the fixation target and cue disappeared and two moving targets appeared. The monkey's task was to track the target that appeared in the cued location. Target color, target direction, distractor condition, and target/ distractor hemifield were all randomized from trial to trial, as in the color-cue task. The initial fixation target never changed color, and the cue was always presented in a valid location.

tionary before starting to move) with the original color cue experiment (targets are initially moving). Specifically, because the targets were slightly brighter than the background, the appearance of the targets was accompanied by an increase in the local luminance of the display. In the original color-cue experiment, this luminance transient occured at the same time as the target motion. However, this was not the case in the "delayed-motion" experiment. In the "spatial-cue" experiment, the luminance transient was coincident with target motion and yet the latencies were comparable to the "delayed-motion" experiment. This indicates that it was not the luminance transient that was responsible for the longer latencies in the original experiment, but rather the greater uncertainty in initial target position.

The results of the last two experiments support the idea that there is a visual search stage involved in smooth pursuit target selection, and that this stage necessarily precedes pursuit initiation, but only when target and distractor are moving in opposite directions. When target and distractor are moving in the same direction, visual search may proceed in parallel with pursuit initiation and need not extend the latency of pursuit. In this case, the results of the visual search are expressed at the time the animal makes a vertical saccade to foveate the chosen target. However, visual search does not fully account for the dependence of latency on distractor condition. On the contrary, the direction of the distractor relative to the target produces a characteristic pattern of latencies regardless of how the animal is cued and even when up to $800 \mathrm{msec}$ is allowed for visual search. Our results indicate that the visual search stage takes up about $25 \mathrm{msec}$, and this only accounts for about half of the extended latency found when target and distractor move in opposite directions. Visual search does not account at all for the fact that latencies are significantly reduced when target and distractor move in the same direction.

\section{The role of expectation}

In the previous experiments, several key variables were randomized from trial to trial so that the animal would not be able to predict the color, location, or direction of the target. It is possible that the ability of the distractor to influence pursuit initiation depends on the uncertainty introduced by this randomization. This uncertainty forces the animal to monitor several possible target locations and be prepared to track either color target moving in either direction. We therefore did a control experiment without randomization so that on each trial the same color target was presented starting from the same position and moving in the same direction. In particular, we used the green square starting in the lower left position and moving to the right (towards the vertical meridian). This target was presented by itself on onethird of the trials, paired with a red distractor moving in the same direction on one-third of the trials, and paired with a red distractor moving in the opposite direction on the remaining third of the trials. Each experiment lasted a minimum of 350 total trials. Because the target motion was the same on all trials, it was possible that the results might be confounded by anticipatory eye movements. We therefore did the experiment both with and without catch trials, and obtained essentially the same results in each case.

The reduced version of the color cue task is directly comparable to the "delayed-motion" and "spatial-cue" variants reported above. In all three experiments, there was no need for the animal to attend to the distractor at the time the target began to move. Nevertheless, the distractor continued to exert an influence on the latency of pursuit. In fact, for the "opposite" condition, pursuit latencies were quantitatively indistinguishable from the "delayed-motion" and "spatial-cue" experiments (Fig. 9 and Table 1, "expectation"). For the "single" and "same" conditions, latencies were slightly longer than in the prior three experiments. This can probably be attributed to a lack of vigilance on the part of the monkey owing to the repetitive nature of the task, rather than some form of adaptation. For one monkey (F), latencies for all distractor conditions gradually lengthened during the course of the session; latencies for the first 50 presentations of each trial type averaged about $10 \mathrm{msec}$ shorter than for the following 80 presentations. This only happened when there were no catch trials and responses could be more automatic. When catch trials were introduced, his performance was stable for the duration of the session. Monkey B showed no such trend either with or without catch trials.

\section{Probability summation}

A somewhat curious result of the preceding experiments is that pairing a pursuit target with a distractor moving in the same direction decreases the latency of pursuit by about $15 \mathrm{msec}$ rel- 


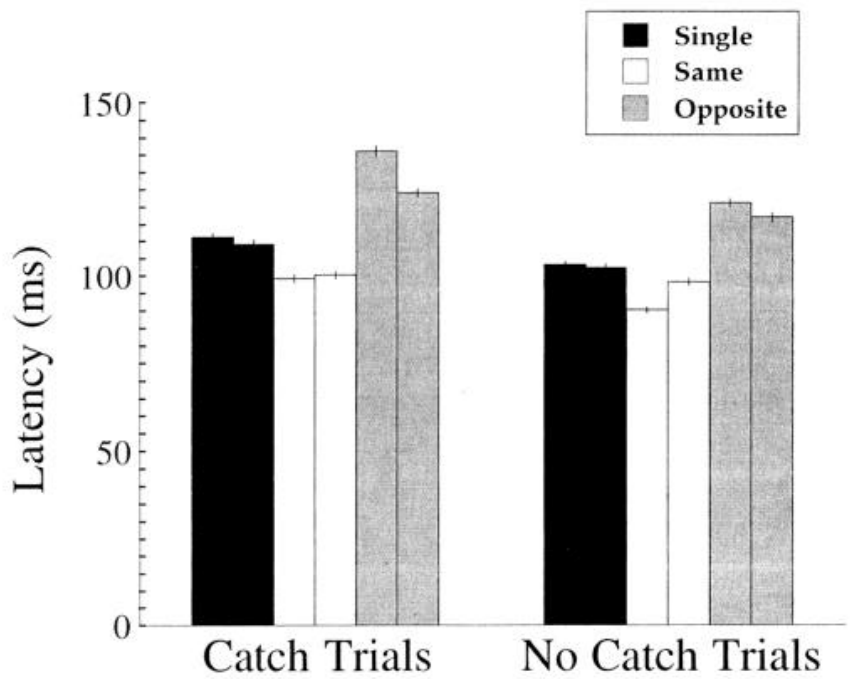

Figure 9. Smooth pursuit latencies for the "expectation" control. The SOA for all conditions was $250 \mathrm{msec}$. The target was always a green square that appeared in the lower left quadrant and moved toward the vertical meridian. The target was presented without a distractor on 1/3 of the trials (black bars), with a distractor moving in the same direction on $1 / 3$ of the trials (open bars) and with a distractor moving in the opposite direction on the remaining 1/3 of the trials (shaded bars).

ative to a single target. In this case, horizontal smooth pursuit may be initiated before a target is selected, thus blurring the distinction between target and distractor. The real issue is how two discrete targets separated by $6.0^{\circ}$ or more combine to produce shorter latencies than a single target. One possibility is that two targets present a larger effective sensory stimulus, possibly because they generate a stronger response motion detectors in the pursuit pathway with large receptive fields. We tested this by measuring latencies for single targets whose linear dimensions were doubled (thus quadrupling the area). Smooth pursuit latencies for large targets (Table 1, first row, numbers in parentheses) were, in fact, slightly longer than latencies for small targets. Thus, simple spatial summation (linear or areal) does not appear to account for the short latencies found when target and distractor moved in the same direction.

Another possibility is that the two targets are processed independently, perhaps by neurons with separate or partially overlapping receptive fields, and that either independent process may produce an eye movement. If the response processes are statistically independent, the behavioral latency should be governed by the laws of probability summation. If we call the two processes A and B and assume that the latency of each process follows the same normal distribution with mean $T$ and standard deviation $\sigma$, then the probability, $P(t)$, of a behavioral response as a function of time after target motion onset is the probability that the latency of $\mathrm{A}$ is equal to $t$ and that the latency of $\mathrm{B}$ is longer than $t$ :

$$
P(t)=\frac{2}{\pi \sigma^{2}} \exp \left(\frac{-(t-T)^{2}}{2 \sigma^{2}}\right) \int_{t}^{\infty} \exp \left(\frac{-(\tau-T)^{2}}{2 \sigma^{2}}\right) d \tau
$$

The mean, $T^{\prime}$, of the resulting probability distribution, $P(t)$, is given by

$$
T^{\prime}=T-\frac{\sigma}{2} .
$$

If we take our estimates of $T$ and $\sigma$ from the latency distribution

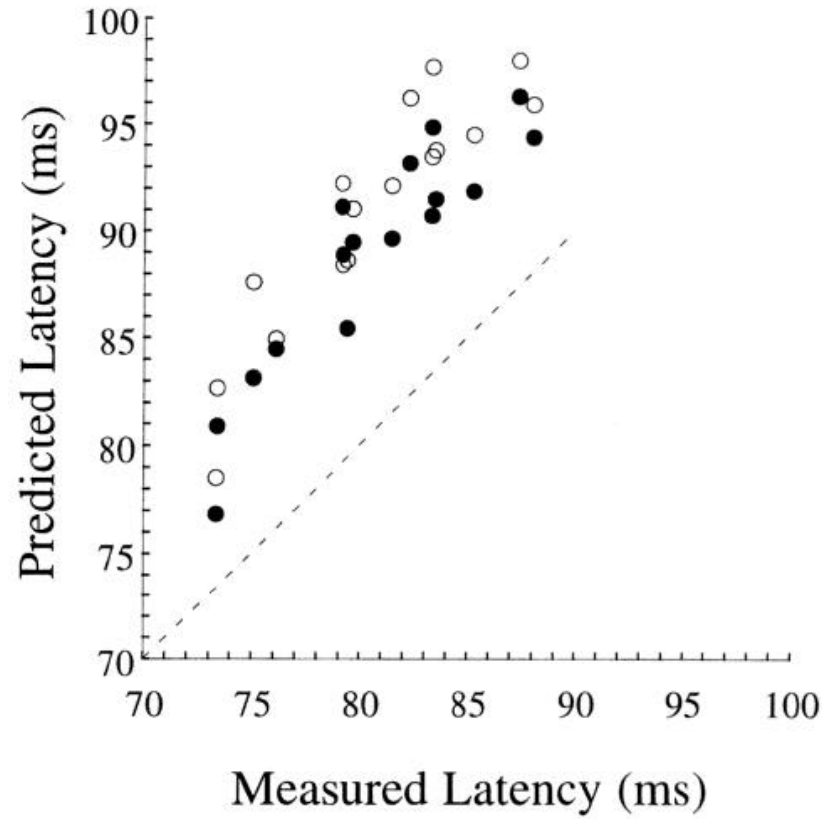

Figure 10. An attempt to predict smooth pursuit latencies for target and distractor moving in the same direction by assuming probability summation of the latencies for single targets. The data shown are for two monkeys, two directions of motion, and four SOAs (16 conditions overall). The task was the original color cue in which the targets were moving when they appeared. The abscissa is the measured latency for target and distractor moving in the same direction, while the ordinate is the latency predicted on the basis of (1) a Gaussian distribution of single target pursuit latencies (open circles), or (2) a resampling of the single target latency distribution (closed circles). The dashed line is where the predicted and measured latencies are equal.

for single targets and if this distribution is approximately Gaussian, then we can make exact predictions about the expected latency for two targets moving in the same direction. We carried out these calculations using the mean and SD for single target latency distributions taken from the original color cue experiment. The predictions are shown as the open circles in Figure 10. Each data point is the measured versus predicted latency for a particular monkey, SOA, and horizontal target direction, but includes different target colors and vertical positions.

On the other hand, if the single target probability distribution is not Gaussian, then we can estimate the expected latency for two targets with a simple empirical procedure: we draw two samples at random from the single target latency distribution and throw out the larger number, keeping the smaller number. By repeating this sampling procedure a number of times, we arrive at the expected latency distribution for two targets. The means of the resampled distributions are plotted as the closed symbols in Figure 10.

The results indicate that probability summation may result in some time savings. However, neither set of predictions in Figure 10 fully accounts for the measured latencies. The mean latencies for two targets moving in the same direction are always shorter that those predicted by either the theoretical (Gaussian) or resampled distributions. When one plots out the actual latency distributions (Fig. 11), it is clear that not only are the single target latency distributions non-Gaussian, but there is also a substantial number of two-target latencies that are shorter than the shortest single target latency. Thus, no amount of resampling can generate the two-target distribution from the single-target 


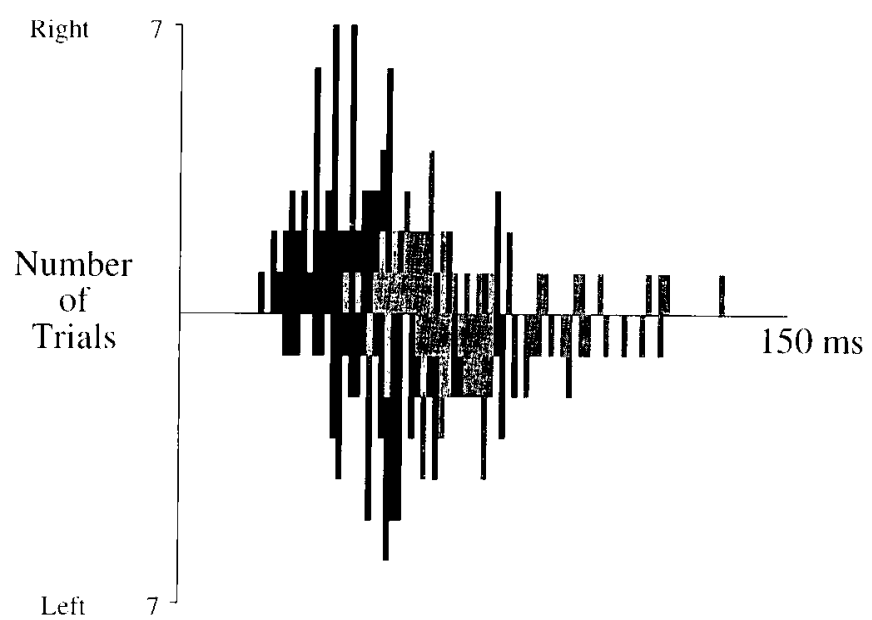

Figure 11. The actual latency distributions for a single monkey at a single SOA upon which the some of the predictions in Figure 10 were based. The dark histograms are latencies for target and distractor moving in same direction while the light histograms are latencies for single targets.

distribution. This suggests that the processes or sets of neurons that respond to each target are not independent, but interact in a cooperative manner. This feature will be incorporated into a simple network model to be developed in the next section.

\section{Discussion}

\section{Competitive network model of the sensorimotor bottleneck}

It has been suggested that selective attention might be implemented as a competitive or "winner-take-all" network (Feldman and Ballard, 1982; Koch and Ullman, 1985). In such a network, units that represent competing inputs mutually inhibit one another. Units that represent similar inputs may interact cooperatively through mutual excitation. Such networks have been characterized analytically (Wilson and Cowan, 1972) and have been used to model various phenomena in hinocular stereo and motion psychophysics (Marr and Poggio, 1976, 1978; Marr et al., 1979; Williams et al., 1986; Williams and Phillips, 1987; Wilson et al., 1992). We will now present a simple competitive network as a model for the sensorimotor bottleneck in smooth pursuit. This network has two properties that correspond closely to the behavior that we have observed. First, the network responds selectively, in an all-or-none manner, to one of its inputs rather than responding in a graded fashion to some vectorial combination of its inputs. This property corresponds to the behavioral observation that eye acceleration is not affected by the motion of the distractor, so that the response, when it occurs, appears to be driven solely by the selected input. Second, the time it takes the network to begin to converge on a stable output depends on the motion of the distractor in a manner that parallels the behavioral latency pattern in our monkeys. This time is shortest when the inputs cooperate (target and distractor moving in same direction, and longest when the inputs compete (target and distractor moving in opposite directions).

The model consists of four excitatory neurons plus four inhibitory interneurons; only the excitatory neurons receive direct input. Figure 12 shows the excitatory units and their effective connections. The actual connections of all the excitatory and inhibitory units in the model can be derived from the equations listed below. Two of the excitatory units respond to rightward motion and the other two respond to leftward motion. The reason

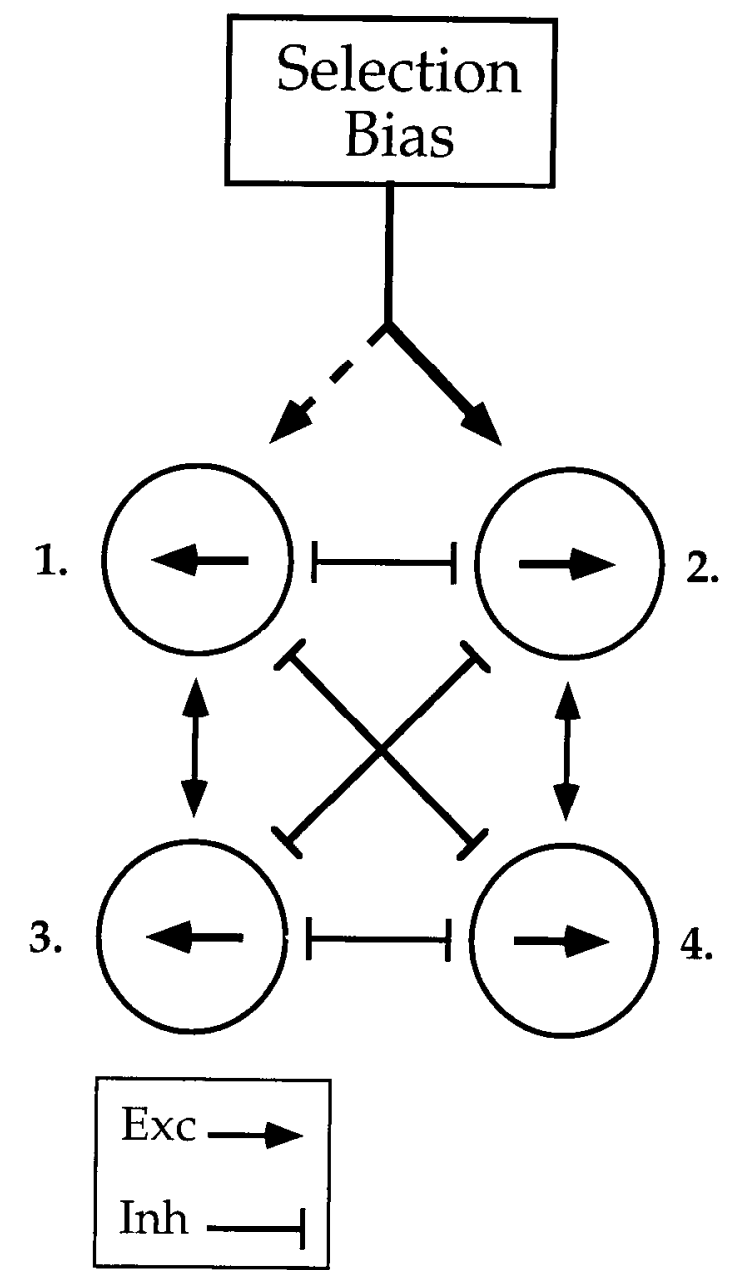

Figure 12. Schematic diagram of competitive network model. This diagram shows only the excitatory units and their effective connections. Arrowheads indicate excitatory connections. Bars indicate inhibitory connections. The selection bias is shown favoring rightward motion.

that there are two units for each direction rather than just a single unit is to allow for some degree of cooperative interaction between a target and a distractor moving in the same direction. The two units for each direction may be thought of as having receptive fields centered at different locations. It is an open question as to whether the units in the model represent competing stimuli, response processes, or some intermediate stage of processing.

In addition to sensory input, each excitatory neuron is capable of receiving a signal that we refer to as the selection bias. The selection bias is meant to represent a cognitive signal related to the animal's choice of targets. Furthermore, we imagine that factors such as anticipation (the animal might guess which way the target will move even though direction is randomized) and expectation (the animal might know which way the target will move because direction is not randomized) will affect the strength of the bias signal. In the model, we simply add the sensory input and bias signals together and provide the sum as a step input starting at time zero. Thus, we tacitly assume that the cognitive signal is available at the same time as the sensory signal. It will be of interest to determine the actual time courses of these signals by recording from individual neurons at various stages of the pursuit pathway.

The equations used to simulate the network were the follow- 

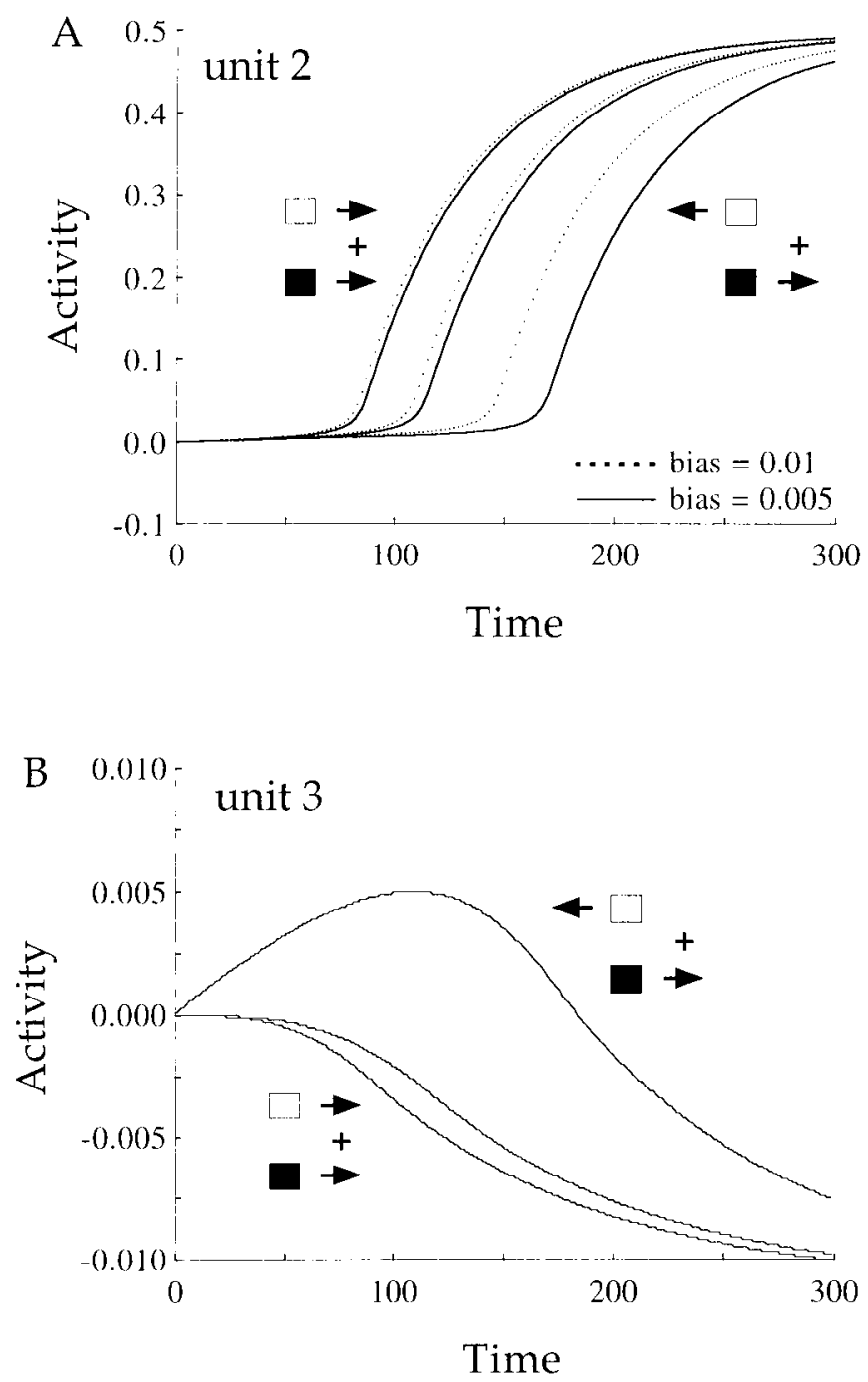

Figure 13. Responses of the model. A, Output of unit 2, which was chosen to represent the target. Solid traces are responses obtained with the weaker bias level, dotted lines are responses obtained with a stronger bias. The response latency is shortest when target (solid square) and distractor (shaded square) move in the same direction and longest when they move in opposite directions. $B$, Response of unit 4 , which signals the direction opposite to that of the target. When there is no distractor, or a distractor that moves in the same direction as the target, this unit receives no excitatory input and is inhibited by the activity of units 2 and 3. When therc is a distractor moving in the opposite direction, the input to unit 4 evokes a small increase in activity, which is later suppressed by inhibition from the more strongly activated units representing the target.

ing (see Wilson and Cowan, 1972). The activity of each excitatory unit $\left(E_{i} ; i=1,2,3,4\right)$ was determined by

$$
\begin{aligned}
\tau \frac{d}{d t} E_{i}(t)= & -E_{i}(t)+\left[1-E_{i}(t)\right] \times \\
& S\left(k\left\{\sum_{j=1}^{N} \beta_{E} E_{j}(t)-\sum_{j=1}^{N} \beta_{i} I_{j}(t)+\text { input }\right\}\right),
\end{aligned}
$$

where $\tau=1.0, k=10.0$, and $\beta_{\mathrm{E}}=10.0$ when the preferred direction of the $i$ th unit was the same as the preferred direction of the $j$ th unit and zero otherwise. $\beta_{1}=10.0$ when the preferred direction of the $i$ th unit was opposite that of the $j$ th unit, and zero otherwise. This scheme for setting the value of the $\beta s$ is
Table 2. Inputs to the network

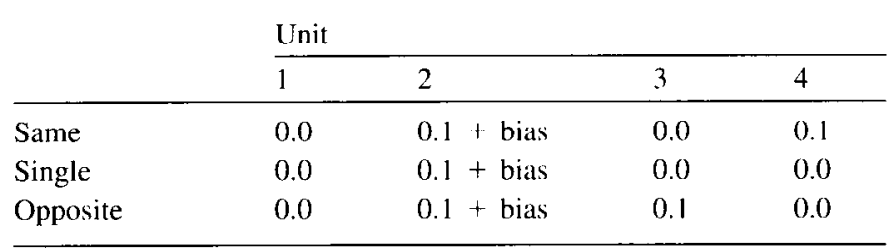

sufficient to ensure that units with the same preferred direction cooperate while those with opposite preferred directions compete. The activity of each inhibitory unit $\left(I_{i}\right)$ was determined in a similar manner, but without the input term:

$$
\begin{aligned}
\tau \frac{d}{d t} I_{i}(t)= & -I_{i}(t)+\left[1-I_{i}(t)\right] \times \\
& S\left(k\left\{\sum_{j=1}^{N} \beta_{E} E_{j}(t)-\sum_{j=1}^{N} \beta_{j} I_{j}(t)\right\}\right) .
\end{aligned}
$$

The imputs to each unit pass through a sigmoidal non-linearity $S(x)$ that prevents unit activity from going to positive or negative infinity. For this compressive non-linearity, we used the logistic function

$$
S(x)=\{1+\exp [-\eta(x-\theta)]\}^{-1}-[1+\exp (-\eta \theta)]{ }^{\prime} .
$$

We performed numerical simulations of this network using a fourth-order Runge-Kutta approximation. The simulations were run on a MC68040-based computer. As a convention, we selected unit 2 (see Fig. 12) to represent the motion of the target and units 3 or 4 to represent the distractor. Figure $13 \mathrm{~A}$ shows the activity of unit 2 during six simulated runs of the network (three distractor conditions $x$ two bias levels). For ease of reference, the pattern of inputs for each of the simulations is given by Table 2 .

In general, the activity of unit 4 was almost identical to that of 2 , while the activities of units 1 and 3 remained close to zero (Fig. 13B). For the first three runs, the bias level was 0.05 . The response to the target (Fig. 13A, heavy lines) is shifted in time by an amount that depends on the motion of the distractor, but the shape of the response does not vary. Figure $13 B$ shows the response of unit 3 , the unit that signals the direction opposite to that of the target (note that the scale of the ordinate is 1/30 that of Fig. 13A). For the "same" and "single" conditions, this unit receives no excitatory input, only inhibition from units 2 and 4 . However, in the "opposite" condition, unit 3 receives a direct input, resulting in a small activation that is enough to delay the onset of the response of unit 2 to the target.

When the monkey is able to predict the motion of the target, we postulate that there is a cognitive expectation that increases the strength of the bias signal. To see what effect this would have on the performance of the network, we did a second set of runs in which we doubled the bias signal to 0.01 (Fig. 13A, dotted lines). The stronger bias substantially shortened the latency of the response in the "opposite" condition, but had little effect on the response in the "same" and "single" conditions. This might explain the pattern of results we observed in the "delayed-motion," "spatial-cue," and "expectation" experiments. While the absolute differences in response timing for the three distractor conditions may shift depending on the strength of the bias signal, the relative order is always the same. This is the same pattern that is revealed by our behavioral experiments 
and we believe it to be a defining characteristic of the sensorimotor bottleneck.

\section{General discussion}

The selection of appropriate targets for goal-directed movements is an important issue in sensorimotor integration. The smooth pursuit system is a sensorimotor pathway in the primate that generates a smooth eye movement in response to a small moving target. In this study, we have sought to describe the behavior of the pursuit system when it is faced with a simple decision about which of two moving targets to use as its input. We studied this hehavior by training rhesus monkeys to choose hetween two simultaneously presented pursuit targets. The two targets were distinguished by their color, and the monkeys task was to track the target that matched the color of a previously presented cue. We have used the term "distractor" to refer to the nonmatching target. We found that the motion of the distractor has a powerful effect on the latency of smooth pursuit such that the onset of pursuit is delayed by about $50 \mathrm{msec}$ (relative to the latency for a single target) when the target is paired with a distractor moving in the opposite direction. A distractor moving in the same direction as the target shortens the latency of pursuit by about 15 msec. In contrast, we found that the motion of the distractor does not appreciably affect eye acceleration once pursuit has been initiated. In particular, a distractor moving in the opposite dircction as the target docs not reduce cyc acceleration as might be expected if the pursuit response represented some compromise between the motions of the target and distractor. Thus, while the distractor affects the timing of the pursuit response, once the response has started it appears to be driven entirely by the selected input.

We found that the motion of the distractor results in a characteristic pattern of response latencies, and that this pattern was obtained despite a number of manipulations designed to alter the cognitive requirements of the task. First, we found that reducing the spatial uncertainty in the initial target location shortened pursuit latency for the distractor-opposite condition by about 25 msec. Thus, while visual search is an important component of the task, it does not account for the overall pattern of results. Even eliminating all uncertainty about the target including its color and direction did not alter the basic latency pattern. Furthermore, the animal did not need to attend to the motion of the distractor for it to affect pursuit latency. We believe that the most consistent framework for interpreting our results is one in which the target and distractor either compete or cooperate, depending on their relative motions. Competitive models have a long history in motion psychophysics (Wilson and Cowan, 1972; Williams et al., 1986; Williams and Phillips, 1987), including a current model of motion perception where the outputs of different motion sensors must be combined (Wilson et al., 1992) and a model of behavioral performance in a choice-discrimation task based on perceived motion (Salzman and Newsome, 1994). A recent review provides a more expansive treatment of the role of competitive networks in selective visual attention (Desimone and Duncan, 1995).

Our behavioral observations are consistent with a competitive network model because (1) competitive networks respond selectively to one of many inputs rather than averaging (linearly combining) their inputs, and (2) the amount of time it takes for a competitive network to converge on a stable output depends upon whether the inputs compete or cooperate. This competition may be influenced or guided by cognitive factors, but does not depend on the need to attend to the distractor. Cognitive factors such as attention may modulate the performance of the network by changing the strength of a signal that biascs the outcome of the competition. However, the competition may also be driven in a bottom-up manner by perceptual salience (Koch and IJIIman, 1985) so that there is no need to consciously plan every eye movement. Our ideas regarding competitive networks and response selection are similar to those of Koch and Ullman (1985), except that rather than thinking of competition as a mechanism for attention per se, we think of it as an automatic process that is modulated by attention.

A competitive network model is also consistent with behavioral observations regarding target selection for saccades (Ottes et al., 1985; Scheinberg and Zelinski, 1993; Munoz and Corneil. 1995). It has been found that when two saccade targets are close together, animals tend to make averaging saccades that land midway between the two targets. As the angular separation between the targets increases, the proportion of averaging saccades goes down and the proportion of on-larget saccades goes up. Saccade latency also increases with target separation. Thus, saccade targets that are close together appear to invoke cooperating response processes, while those that are far apart invoke competing processes. In the present study, we have not seen any evidence for an averaging process in smooth pursuit. However, our experiments did not include conditions that would be expected to favor "averaging pursuit," i.e., small angular separations between target directions or small differences in speed.

The idea that cognitive decisions might exert their influence by shifting the balance between competing processes or states may help to explain other behavioral aspects of the pursuit system. For example, the decision to initiate pursuit could be implemented by shifting the balance between a fixation state and a movement state. Previous work in our lab (Schwartz and Lisberger, 1994) has demonstrated that there is, indeed, an energy barrier separating pursuit from fixation as evidenced by the observation that the gain of the eye-movement response to a small movement of the larget is much less when the animal is fixating than when he is tracking. This is what one would expect if the fixation and movement processes are each self-reinforcing. Similar ideas have been put forth regarding the initiation of saccadic eye movements and, indeed, a "fixation system" has been identified for saccades that involves the posterior parietal cortex and rostral pole of the superior colliculus (Posner et al., 1984; Munoz. and Wurtz, 1993). It would be of interest to know if the saccade and pursuit systems, which are largely segregated in other respects, share the same fixation system.

In a sense, the distinction between target and distractor is similar to the distinction between target and background, particularly when there are many distractors. However, the effect on smooth pursuit of a moving distractor is much different than the effect of a stationary textured background. Kimmig et al. (1992) found that a stationary background reduced the initial eye acceleration, but did not affect the latency of pursuit, which is the converse of the pattern of results found with a small moving distractor. It should be noted that our experiments were performed with room illumination roughly equal to the background luminance of the video display $\left(10 \mathrm{~cd} / \mathrm{m}^{2}\right)$, so that the full visual scene, comprising surfaces of many different textures, colors, and depths, was clearly visible. Kimmig et al. found that the effect of the background was reduced when the target was presented in a different depth plane. It would be interesting to know 
if the effects found in this study depend on the relative depth of the target and distractor.

We bclieve that the general framework of a competitive network modulated by cognitive processes will be useful for further studies on the neural basis of target selection. For example, it raises questions about where the competition takes place and how it is implemented. There are two extrastriate areas in visual cortex, MT (middle temporal) and MST (medial superior temporal), that are thought to play a key role in the pursuit pathway due to (1) a preponderance of direction selective neurons, (2) direct anatomical projections of these areas to brainstem nuclei known to be involved in pursuit, and (3) deficits in smooth pursuit following lesions of either area. There is already evidence for inhibitory interactions among cells signalling opposite directions of motion in MT and MST (Snowden et al., 1991; Qian and Andersen, 1994). These studies found that the response of some MT and MST neurons to a field of random dots moving in the preferred direction was reduced when other dots moving in the null direction were also present. However, it is unlikely that the suppression seen in MT and MST can fully explain the effect of a distractor on pursuit latency. First, this suppression has not been demonstrated in cases where the two stimuli are separated by $6^{\circ}$ or more, as in the present experiments. In fact, we found that the effect of the distractor is equally strong when the target and distractor are in the same or opposite hemifields. MT cells tend to have receptive fields that are limited to the contralateral hemifield and their response is not likely to be suppressed by the motion of a distractor in the opposite hemifield. Second, the suppression hypothesis does not account for the facilitation provided by a distractor moving in the same direction. Third, the behavior shows complete selectivity for one input or the other, which would require complete suppression of the distractor motion. Complete suppression is rarely seen in MT or MST. Fourth, in the two studies cited, it was found that opposing motion reduced the amplitude of neuronal responses but did not appreciably affect neuronal latency. Thus, the suppression hypothesis requires that there should be a relationship between neuronal response amplitude and behavioral latency, which is not unreasonable. However, another group (Kawano et al., 1994) has recently shown that for ocular following, there is a very precise relationship between eye movement latency and neuronal response latency in MST. Neuronal response amplitude appeared to be correlated with cyc velocity. These considerations make it seem more likely that response suppression in MT or MST would reduce eye acceleration rather than increasing latency. This pattern of results does not fit with our behavioral observations.

In conclusion, we have studied the behavior of the smooth pursuit eye movement system of the primate under conditions where it must decide between two potential moving targets. The response latency of the system shows a characteristic pattern that depends on the motion of the distractor regardless of whether or not there is a need for the monkey to attend to the distractor. The pattern of results is entirely consistent with a competitive network that is modulated by cognitive factors. We believe that this pattern of behavioral results is a defining characteristic of the sensorimotor bottleneck for smooth pursuit and that it will serve as a valuable guide for neurophysiological investigations aimed at localizing the sensorimotor bottleneck and understanding its neural implementation.

\section{References}

Albright TD (1984) Direction and orientation selectivity of neurons in visual area MT of the macaque. J Neurophysiol 52:1106-1130.

Andersen RA (1987) Inferior parietal lobule function in spatial perception and visuomotor integration. In: Handbook of physiology, pp 483-518. Baltimore: Williams \& Wilkins.

Brodal P (1978) The corticopontine projection in the rhesus monkey: origin and principles of organization. Brain 101:251-283.

Brodal P (1979) The pontocerebellar projection in the rhesus monkey: an experimental study with retrograde axonal transport of horseradish peroxidase. Neuroscience 4:193-208.

Brodal P (1982) Further observations on the cerebellar projections from the pontine nucleus and the nucleus reticularis pontis in the rhesus monkey. J Comp Neurol 204:44 55

Bruce CJ, Goldberg ME, Bushnell MC, Stanton GB (1985) Primate frontal eye fields. II. Physiological and anatomical correlates of electrically cvoked eye movements. J Neurophysiol 54:714-7.34.

Desimone R, Duncan J (1995) Neural mechanisms of selective visual attention. Annu Rev Neurosci 18:193-222.

Desimone R, Schein SJ (1987) Visual properties of neurons in area V4 of the macaque: sensitivity to stimulus form. J Neurophysiol 57:835868.

Desimone R, Schein SJ, Moran J, Ungerleider LG (1985) Contour, color and shape analysis beyond the striate cortex. Vision Res 25: $441-452$.

Dursteler MR, Wurtz RH (1988) Pursuit and optokinetic deficits following chemical lesions of cortical areas MT and MST. J Neurophysiol 60:940-965.

Dursteler MR, Wurtz RH, Newsome WT (1987) Directional pursuit deficits following lesions of the foveal representation within the superior temporal sulcus of the macaque monkey. J Neurophysiol 57 $1262-1287$.

Feldman JA, Ballard D (1982) Connectionist models and their properties. Cogn Sci 46:27-39.

Fries W (1981) The projection from striate and prestriate visual cortex onto the pontine nuclei in the macaque monkey. Soc Neurosci Abstr $7: 762$.

Gerrits NM, Voogd J (1989) The topographical organization of climbing and mossy fiber afferents in the flocculus and ventral paraflocculus in the rabbit, cat, and monkey. Exp Brain Res (Suppl) 17:2629.

Glickstein M, Cohen II, Dixon B, Gibson A, Hollins M. LaBossiere $\mathrm{E}$, Robinson $\mathrm{F}$ (1980) Corticopontine visual projections in macaque monkeys. J Comp Neurol 190:209-229.

Glickstein M, May J, Mercer BE (1985) Corticopontine projection in the macaque: the distribution of labeled cortical cells after large injections of horseradish peroxidase in the pontine nuclei. J Comp Neurol 235:343-359.

Goodale MA, Milner AD (1992) Separate pathways for perception and action. Trends Neurosci 15:20-25.

Gross CG, Rocha-Miranda CE, Bender DB (1972) Visual properties of neurons in inferotemporal cortex of the macaque. $\mathbf{J}$ Neurophysiol 35 : 96-111.

Judge SJ, Richmond BJ, Chu FC (1980) Implantation of magnetic search coils for measurement of eye position. Vision Res 20:535538 .

Kawano K, Shidara M, Watanabe Y, Yamane S (1994) Neural activity in cortical area MST of alert monkey during ocular following responses. J Neurophysiol 71:2305 2324.

Keating EG (1991) Frontal eye field lesions impair predictive and visually-guided pursuit eye movements. Exp Brain Res 86:311-32.3.

Kimmig HG, Miles FA, Schwarz U (1992) Effects of stationary textured backgrounds on the initiation of pursuit eye movements in monkeys. J Neurophysiol 68:2147-2164.

Koch C, Ullman S (1985) Shifts in selective attention: towards the underlying neural circuitry. Hum Neurobiol 4:219-227.

Krauzlis RJ, Lisberger SG (1994) Temporal properties of visual motion signals for the initiation of smooth pursuit eye movements in monkeys. J Neurophysiol 72:150-162.

Lisherger SG, Westbrook I.F (1985) Properties of visual inputs that initiate horizontal smooth pursuit eye movements in monkeys. J Neurosci 5:1662-1673.

Lisberger SG, Morris EJ, Tychsen L (1987) Visual motion processing and sensory-motor integration for smooth pursuit eye movements. Annu Rev Neurosci 10:97-129. 
Lynch JC (1987) Frontal eye field lesions in monkeys disrupt pursuit Exp Brain Res 68:437-441.

MacAvoy MG, Gottleib JP, Bruce CJ (1991) Smooth pursuit eye movement representation in the primate frontal eye field. Cereb Cortex 1:95-102.

Marr D, Poggio T (1976) Cooperative computation of stereo disparity. Science 194:283-287.

Marr D, Poggio T (1979) A computational theory of human stereo vision. Proc R Soc Lond [Biol] 204:301-328.

Marr D, Palm G, Poggio T (1978) Analysis of a cooperative stereo algorithm. Biol Cybern 28:223-239.

Maunsell JHR, Van Essen DC (1983) Functional properties of neurons in the middle temporal visual area (MT) of the macaque monkey: $I$. Selectivity for stimulus direction, speed and orientation. J Neurophysiol 49:1127-1147.

Miles FA, Eighmy BB (1980) Long-term adaptive changes in the primate vestibuln-ocular reflex. I. Rehavioral observations. J Neurophysiol 43:1406-1425.

Munoz DP, Corneil BD (1995) Evidence for interactions between target selection and visual fixation for saccade generation in humans. Exp Brain Res 103:168-173.

Munoz DP, Wurtz RH (1993) Fixation cells in monkey superior colliculus. II. Reversible activation and deactivation. $\mathbf{J}$ Neurophysiol 70 : 576-589.

Mustari MJ, Fuchs AF, Wallman J (1988) Response properties of dorsolateral pontine units during smooth pursuit in the rhesus macaque J Neurophysiol 60:664-686.

Newsome WT, Wurtz RH, Dursteler MR, Mikami A (1985) Deficits in visual motion processing following ibotenic acid lesions of the middle temporal visual area of the macaque monkey. J Neurosci 5:825840 .

Newsome WT, Wurtz RH, Komatsu H (1988) Relation of cortical areas MT and MST to pursuit eye movements. II. Differentiation of retinal from extrarelinal inputs. J Neurophysiol 60:604-620.

Ottes FP, Van Gisbergen JAM, Eggermont JJ (1985) Latency dependence of colour-based target vs nontarget discrimination by the saccadic system. Vision Res 25:849-862.

Posner MI, Walker JA, Friedrich FJ, Rafal RD (1984) Effects of parietal injury on covert orienting of visual attention. J Neurosci $4: 1863-1874$

Qian N, Andersen RA (1994) Transparent motion perception as detection of unbalanced motion signals. II. Physiology. J Neurosci 14: $7367-7380$

Rashbass C (1961) The relationship between saccadic and smooth tracking eye movements. J Physiol (Lond) 159:326-338.

Salzman CD, Newsome WT (1994) Neural mechanisms for forming a perceptual decision. Science 264:231-237.

Schein SJ, Desimone R (1990) Spectral properties of V4 neurons in the macaque. J Neurosci 10:3369-3389.
Schwartz JD, Lisberger SG (1994) Initial tracking conditions modulate the gain of visuo-motor transmission for smooth pursuit eye movements in monkeys. Vis Neurosci 11:411-424.

Scheinberg DL, Zelinsky GJ (1993) A cortico-collicular model of saccadic target selection. In: Perception and cognition: advances in eye movement research (d'Ydewalle G, Van Rensbergen J, eds), pp 334. 348. Amsterdam: Elsevier.

Snowden RJ, Treue S, Erickson RG, Andersen RA (1991) The response of area MT and V1 ncurons to transparent motion. J Neurosci 11: $2768-2785$.

Stone LS, Lisberger SG (1990) Visual responses of Purkinje cells in the cerebellar floccullus during smooth-pursuit eye movements in monkeys. I. Simple spikes. J Neurophysiol 63:1241-1261

Suzuki DA, Keller EL (1988) Visual signals in the dorsolateral pontine nucleus of the alert monkey: their relationship to smooth-pursuit eye movements. Exp Brain Res 53:473-478.

Suzuki DA, May J, Keller EL (1988) Smooth-pursuit eye movement deficits with chemical lesions in the dorsolateral pontine nucleus of the monkey. J Neurophysiol 59:952-977.

Tanaka M, Weber H, Creutzfeldt OD (1986) Visual properties and spatial distribution of neurones in the visual association area on the prelunate gyrus of the awake monkey. Exp Brain Res 65:11-37.

Thiers P, Koehler W, Buettner UW (1988) Neuronal activity in the dorsolateral pontine nucleus of the alert monkey modulated by visual stimuli and eye movements. Brain Res 72:496-512.

Treisman AM (1977) Focused attention in the perception and retrieval of multidimensional stimuli. Percept Psychophysiol 22:1-11.

Treisman AM, Gelade G (1980) A feature-integration theory of attention. Cogn Psychol 12:97-136.

Ungerleider LG, Mishkin M (1982) Two cortical visual systems. In: Analysis of visual behavior (Ingle DJ, Goodale MA, Mansfield RJW, eds), pp 549-586. Cambridge, MA: MIT Press.

Williams D, Phillips G (1987) Cooperative phenomena in the perception of motion direction. J Opt Soc Am [A] 4:878-885.

Williams D, Phillips G, Sekuler R (1986) Hysteresis in the perception of motion direction as evidence for neural cooperativity. Nature 324: 253-255.

Wilson HR, Cowan JD (1972) Excitatory and inhibitory interactions in localized populations of model neurons. Biophys $\mathbf{J}$ 12:1-24

Wilson HR, Ferrera VP, Yo C (1992) A psychophysically motivated model for two-dimensional motion perception. Vis Neurosci 9:79_ 91.

Wurtz RH (1969) Visual receptive fields of striate cortex neurons in awake monkeys. J Neurophysiol 32:727-742.

Zee DS, Yamazaki A, Butler PH, Gucer G (1981) Effects of ablation of the flocculus and paraflocculus on eye movements in the primate. J Neurophysiol 46:878-899.

Zeki SM (1978) Uniformity and diversity of structure and function in rhesus monkey prestriate visual cortex. J Physiol (Lond) 277:27.3290. 